\title{
Application of Calorimetric Low-Temperature Detectors for the Investigation of Z-Yield Distributions of Fission Fragments
}

\author{
Santwana Dubey ${ }^{1,2,{ }^{*}}$, Artur Echler ${ }^{1,2}$, Peter Egelhof $^{1,2}$, Patrick Grabitz ${ }^{1,2}$, Manfred Mutterer ${ }^{1}$, \\ Werner Lauterfeld ${ }^{2}$, Stefan Stolte ${ }^{2}$, Aurelien Blanc ${ }^{3}$, Ulli Köster ${ }^{3}$, Saskia Kraft-Bermuth ${ }^{4}$, \\ Pascal Scholz ${ }^{4}$, Shawn Bishop ${ }^{5}$, Jose Gomez ${ }^{5}$, and Friedrich Gönnenwein ${ }^{6}$ \\ ${ }^{1}$ GSI Helmholtzzentrum für Schwerionenforschung GmbH, 64291 Darmstadt, Germany \\ ${ }^{2}$ Institut für Physik der Universität Mainz, 55128 Mainz, Germany \\ ${ }^{3}$ Institut Laue-Langevin, 38042 Grenoble Cedex 9, France \\ ${ }^{4}$ Institut für Atom- und Molekülphysik, Justus-Liebig-Universität, 35392 Giessen, Germany \\ ${ }^{5}$ Physik-Department, Technische Universität München, 85748 Garching, Germany \\ ${ }^{6}$ Physikalisches Institut, Eberhard Karl Universität, 72076 Tübingen, Germany
}

\begin{abstract}
In recent experiments, the new concept of calorimetric lowtemperature detectors (CLTDs) was applied for the first time for the investigation of isotopic yields of fission fragments. Fragments from neutron-induced fission sources were mass-separated by the LOHENGRIN spectrometer at the ILL Grenoble and, after passing silicon nitride membranes used as degraders, detected in a CLTD array. The new detector concept of a thermal detector provides a fundamental advantage over conventional ionization-mediated detectors, in particular for heavier particle masses at low energies. Using fissile targets of ${ }^{235} \mathrm{U},{ }^{239} \mathrm{Pu}$ and ${ }^{241} \mathrm{Pu}$, nuclearcharge separation was studied in the mass region $82 \leq \mathrm{A} \leq 139$. For light fragments, the $Z$ resolution matches historically best values with conventional techniques, while for heavier masses substantial improvement was attained. We have gained first LOHENGRIN data on the isotopic yields in the light-mass group of ${ }^{241} \mathrm{Pu}$. Towards mass-symmetry, known Z-yield data were supplemented in the range $\mathrm{A}=110$ to 113 for ${ }^{241} \mathrm{Pu}$ and ${ }^{239} \mathrm{Pu}$. Extended data sets were cumulated for $\mathrm{A}=92$ and 96 due to a recent request from studies on the reactor anti-neutrino spectrum. Furthermore, considerable progress was achieved to extend isotopic yield measurements up to the heavy-mass region, hardly accessible until now.
\end{abstract}

\section{Introduction}

Precise data on the characteristics of fission-fragment yield distributions in terms of mass, nuclear charge, and kinetic energy are of great interest, on the one hand, for a better understanding of the fission process and for verifying fission models, and, on the other hand, in applied fields, e.g. for calculating accumulation and inventory of fission products at

\footnotetext{
* Corresponding author: s.dubey@gsi.de
} 
various stages of the nuclear fuel cycle in a reactor [1,2]. Since more than four decades, the recoil mass spectrometer LOHENGRIN [3], available at the ILL Grenoble, has been a leading instrument for fission fragment studies. Fission fragments emerging from a thin fissile target located close to the high-flux reactor core (i.e., at a thermal neutron flux $\approx 5 \times 10^{14} \mathrm{n} \mathrm{cm}^{-2} \mathrm{~s}^{-1}$ ) are separated according to the chosen ratios $\mathrm{E} / \mathrm{q}$ and $\mathrm{A} / \mathrm{q}(\mathrm{E}=$ kinetic energy, $\mathrm{A}=$ fragment mass, $\mathrm{q}=$ ionic charge) and detected with suitable energy detectors (silicon detectors or ionization chambers). For determining isotopic fragment yields, a fairly universal method is the passive absorber technique exploiting the Z-dependent energy loss of fission fragments in an energy degrader [4]. Due to its perfect mass resolution, LOHENGRIN has contributed more high resolution data sets on mass, nuclear charge and energy distributions than any other method, with the isotopic yields, however, being restricted to the region of light fragment masses [5]. Instead, isotopic yields of specific radioactive isotopes in the heavy mass group were determined by high-resolution gamma spectrometry after mass-separation with LOHENGRIN [6] or, formerly, by radiochemical methods [7].

In the present work, we have applied a new experimental approach to determine isotopic yields at LOHENGRIN by the passive absorber method using an array of calorimetric lowtemperature detectors (CLTDs) instead of an ionization chamber for the residual energy measurement. The advantages of CLTDs over conventional ionization detectors (solid-state $\mathrm{Si}$ or gaseous detectors) are the more complete energy detection, the smaller energy gap of the detected quanta (phonons), and the absence of any entrance window or dead layer, leading to substantial improvements in basic detector properties, as for example energy resolution, energy linearity, detection threshold, etc. [8 - 11].

Furthermore, in a preceding experiment [12], stacks of commercially available silicon nitride (SiN) membranes [13] were tested as degrader material, replacing the previously used Parylene-C plastic foils. Silicon nitride is a modern highly resistant material out of which stable and homogeneous membranes thinner than $100 \mathrm{~nm}$ can be fabricated by lithographic techniques. These membranes are extensively used outside nuclear physics, e.g., as substrates in transmission electron microscopy or ionization-chamber entrance windows for low-energy ERDA (elastic recoils detection analysis) applications [14]. We have applied stacks of up to ten $1000 \mathrm{~nm}$ thick SiN membranes for nuclear-charge determination by energy degradation of fission fragments.

\section{Properties of CLTD's for particle detection}

The basic principle of a CLTD is schematically displayed in Fig. 1 (for details see [8]). Incident particles are stopped in an absorber located in an evacuated volume inside a cryostat. The temperature rise of the absorber due to the thermalization of the particle's kinetic energy is measured by a thermometer. Finally, on a time scale of tens of $\mu \mathrm{s}$, the deposited heat is fed back to a heat sink through thermal coupling. The sensitivity of such detectors is inversely proportional to their heat capacity $\mathrm{C}$. Therefore, detector pixels are small in size and operated at low temperatures $\left(\mathrm{C} \sim \mathrm{T}^{3}\right.$, for insulators and superconductors). As thermometers, thermistors with a strong temperature dependence of their resistance at their low operating temperatures, for example superconducting transition-edge thermometers, are used. Since CLTDs are windowless towards the beam-line and signal generation is not based on ionization, but on phonon formation, thus leading to a more complete energy detection, they 


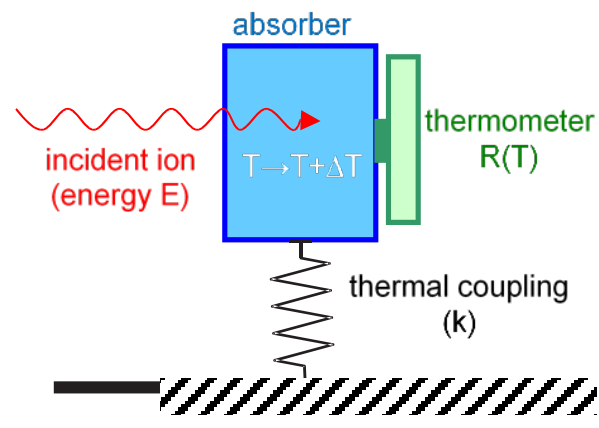

Heat sink

Fig. 1. Principle of particle detection with a calorimetric low temperature detector CLTD (for discussion see text).

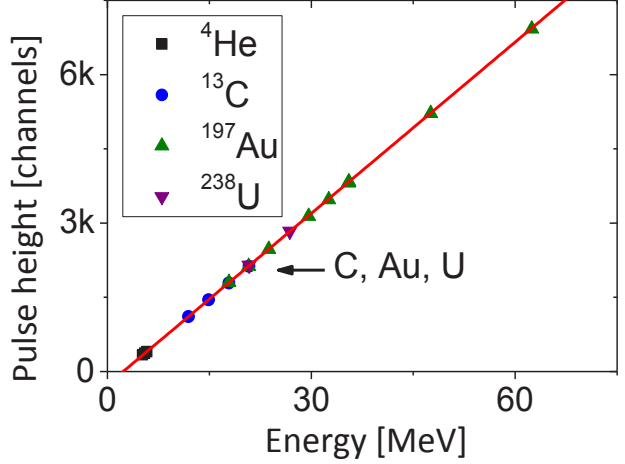

Fig. 2. Systematic study of the linearity of the energy response for various ions and energies. The solid line shows a linear fit to the data [9].

do not experience any pulse-height defect even at high energy loss density. The performance of detector pixels with a layout similar to the present one was systematically investigated in several measurements with various heavy ion beams $\left({ }^{13} \mathrm{C} \ldots{ }^{238} \mathrm{U}\right)$, at incident energies ranging from $0.1 \mathrm{MeV} / \mathrm{u}$ up to $360 \mathrm{MeV} / \mathrm{u}$ [8]. For a wide range of ion species, output signal amplitudes are perfectly linear with energy, and no pulse-height defect is observed (see Fig. 2). Note that even at a quite low energy of $20 \mathrm{MeV}$ a heavy nucleus such as ${ }^{238} \mathrm{U}$ generates the same signal amplitude as the light ${ }^{13} \mathrm{C}$. At the same time, due to the good quantum statistics of phonon formation, and due to the more complete energy detection, the energy resolution is down to $\Delta \mathrm{E} / \mathrm{E}=2-6 \times 10^{-3}$ (FWHM) for heavy ions, being not limited by statistical effects but probably due to slight imperfections in coating, bonding and contacting. As an example, an energy resolution of $\Delta \mathrm{E}=91 \mathrm{keV}(\mathrm{FWHM})$ was achieved for $20.7 \mathrm{MeV}$ ${ }^{238} \mathrm{U}$ ions [8], unmatched by any ionization based detector. This unique performance has potential advantage for energy detection of fission fragments for which, to our knowledge, CLTD's were never applied before.

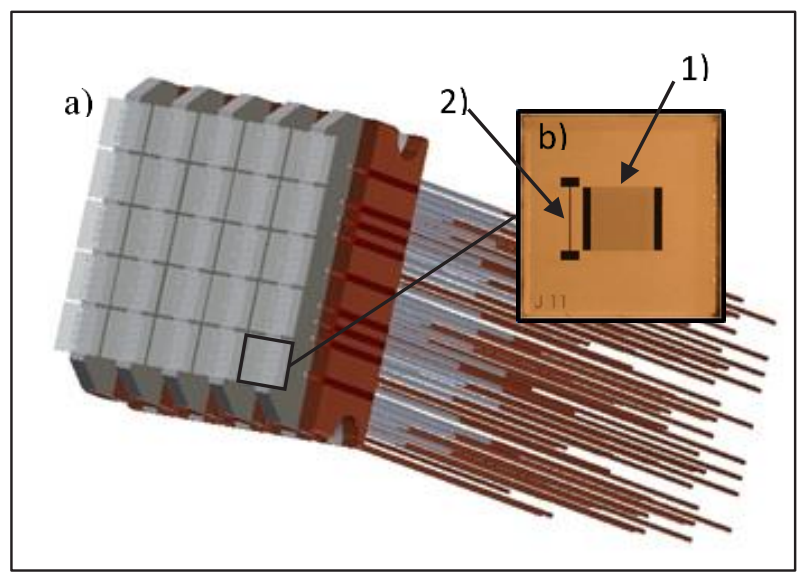

Fig. 3. a) Design of the CLTD-array consisting of $5 \times 5$ detector pixels, b) $3 \times 3 \mathrm{~mm}^{2}$ wide single pixel with Al-transition-edge sensor (1) and Au-heater (2). 
The detector array used in the present experiment consists of 25 pixels with a total active area of $15 \times 15 \mathrm{~mm}^{2}$ (Fig. 3). To operate the array, a windowless pumped ${ }^{4} \mathrm{He}$-bath cryostat is used. An individual pixel is made of a sapphire substrate $\left(\mathrm{Al}_{2} \mathrm{O}_{3}\right)$ serving as absorber with a thickness of $430 \mu \mathrm{m}$ and an area of $3 \times 3 \mathrm{~mm}^{2}$. On its rear side, a $10 \mathrm{~nm}$ thick aluminum thermistor is evaporated as temperature sensor, operated at the phase transition between the normal and superconducting state at $T_{\mathrm{c}}=1.5 \mathrm{~K}$. Proper thermal coupling is provided to a heat sink. The insert in Fig. 3 (Fig. 3b) shows a single pixel, with an additional gold wire strip serving as heater used for temperature stabilization. A weak ${ }^{241} \mathrm{Am} \alpha$ source is facing the array for calibration purposes. For the readout, conventional pulse electronics consisting of low-noise preamplifiers and flash-ADCs are used.

\section{Experimental set-up at LOHENGRIN}

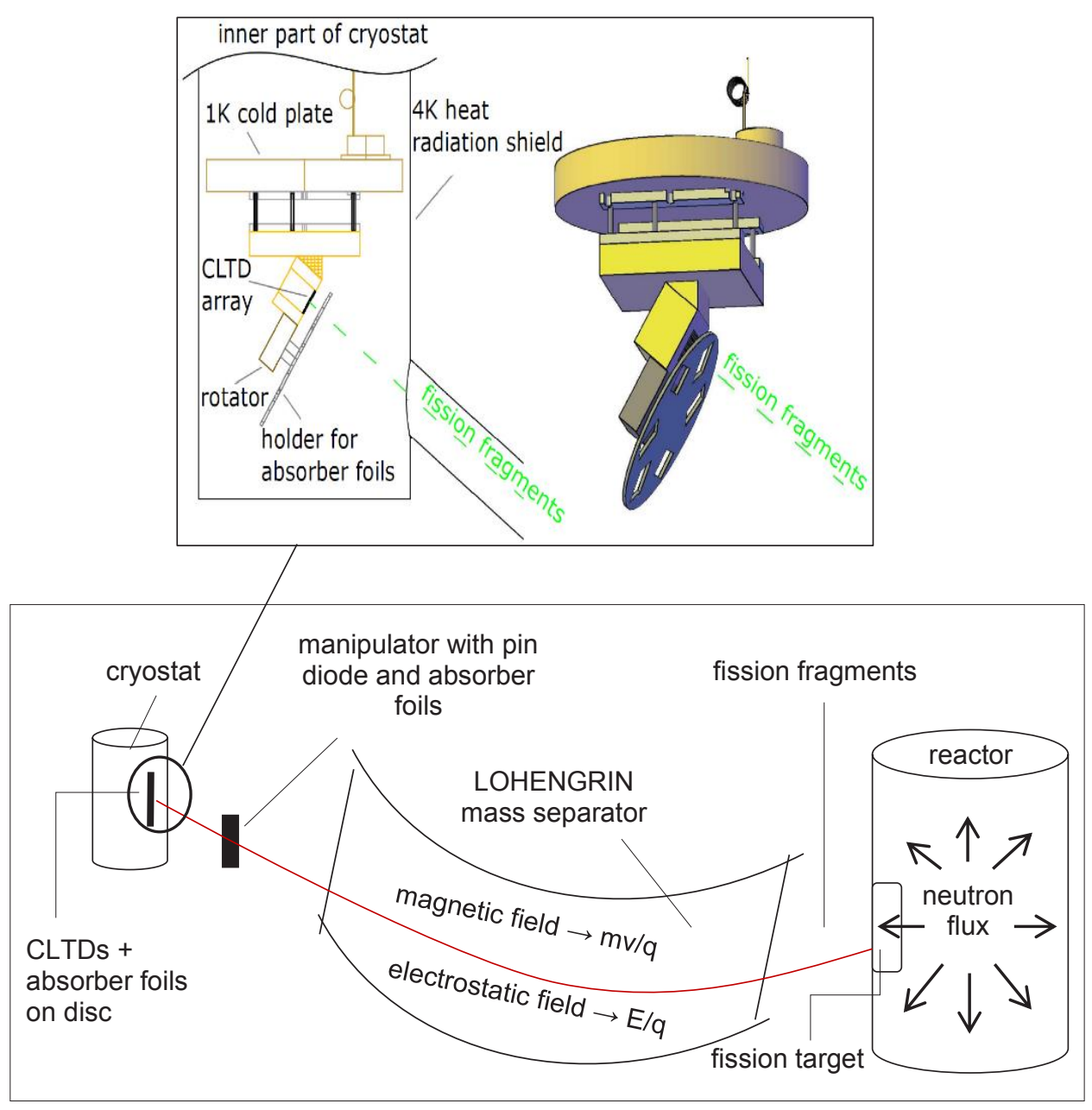

Fig. 4. Schematic view of the LOHENGRIN mass separator with the arrangement of silicon nitride absorber foils and the CLTD's as residual-energy detector. 
The ${ }^{4} \mathrm{He}$-bath cryostat containing the CLTD array of 25 independent detector pixels with a total active area of $15 \times 15 \mathrm{~mm}^{2}$ was coupled, without any entrance foil, to the straight exit flange of LOHENGRIN inclined by $35^{\circ}$ (see Fig. 4). However, the adaption of the cryogenic system to the LOHENGRIN beam line affected the CLTD energy resolution by heat radiation and, accordingly, required an entrance slit of $\sim 200 \mathrm{~mm}^{2}$. In a first attempt [12], SiN absorbers were mounted on a movable manipulator at a distance of $95 \mathrm{~cm}$ outside the cryostat. This resulted in largely reduced counting efficiency due to small-angle scattering and increased the background by contaminating mass lines. To counter these problems, the experimental set-up was upgraded by installing a remotely controlled sample changer for SiN absorber foils inside the cryostat, at a distance of few millimeters to the CLTDs.

The design of the new system is displayed in Fig.4. The rotatable disk, with six positions for $\mathrm{SiN}$ foils of $16 \times 10 \mathrm{~mm}^{2}$ area each, is operated by a remote-controlled piezo-driven rotary stepper positioner. We use the system ANR240/RES from Attocube [15], which consists of a positioner with resistive encoder along with the controller ANC350. The device operates under vacuum and at temperatures as low as $10 \mathrm{mK}$, and allows a reproducible positioning with an accuracy of $0.050^{\circ}(\approx 30 \mu \mathrm{m}$ for the current design $)$. The SiN foil stacks mounted on this disc were $1 \mu \mathrm{m}, 4 \mu \mathrm{m}, 5 \mu \mathrm{m}, 6 \mu \mathrm{m}, 7 \mu \mathrm{m}$ thick. In addition, the CLTD array was tilted perpendicular to the beam, increasing the effective detector area by $20 \%$ as compared to the previous runs. Optionally, some more absorber foils were still mounted onto the manipulator upstream (95 cm away from CLTDs) for varying the total absorber thickness up to $10 \mu \mathrm{m}$, at the cost of reduced transmission. A silicon PIN-diode, which served as a yield monitor for adjusting the LOHENGRIN mass separator, was also mounted on this manipulator.

\section{Experimental results and discussion}

Using fissile targets of ${ }^{235} \mathrm{U},{ }^{239} \mathrm{Pu}$ and ${ }^{241} \mathrm{Pu}$, the quality of nuclear-charge separation was studied for selected masses in the region $82 \leq \mathrm{A} \leq 139$ as a function of degrader thickness and fission-fragment kinetic energies. For the light fragment group, good Z-resolution was obtained (see Fig. 5b), sufficiently high to clearly separate individual nuclear charges in the residual-energy spectra and, as expected, with the resolving power improving towards higher energy losses. We could already match the historically best $Z$-resolving power, e.g. $Z / \Delta Z=$ 55 at $Z=37(\Delta Z$ is defined as the ratio of the peak width in FWHM and the difference between adjacent peaks in the rest-energy spectra [4]) (see Fig. 5a), achieved conventionally with Parylene-C absorbers and ionization chambers $[16,17]$. We have gained first LOHENGRIN data on the isotopic yields in the light-mass group of ${ }^{241} \mathrm{Pu}$ fission for $\mathrm{A}=89$ to 109 . Fragments from ${ }^{241} \mathrm{Pu}\left(\mathrm{n}_{\mathrm{th}}, \mathrm{f}\right)$ were previously studied at the ILL by time-of-flight mass spectrometry with the aid of the Cosi-Fan-Tutte spectrometer [18].

Towards mass symmetry, known Z-yield data were supplemented in the range $A=110$ to 112 for ${ }^{241} \mathrm{Pu}$, and $\mathrm{A}=111$ to 113 for ${ }^{239} \mathrm{Pu}$. The investigation of isotopic yields for the heavier masses $\mathrm{A}>108$ (Fig. 5c) was started with the aim to study the onset of even-odd effects in the transition region from the light fragment group towards the symmetry region, which is of high interest for verification of nuclear models [19]. The data analysis concerning these fragment yields is presently in progress.

For heavy fragments with $\mathrm{A}>128$, the obtained Z-resolution was insufficient to resolve individual peaks in the residual energy spectra. Instead, the individual intensities have to be 
retrieved from constrained fitting of the overlapping peaks (see Fig. 5d), a method well established in high-precision mass spectrometry [20]. In our case, the fitting quality benefits from the outstanding properties of the CLTDs for the low-energy fission fragments after energy degradation (see above).

Preceding test measurements at the tandem accelerator at the MLL Garching with stable ${ }^{130} \mathrm{Te}$ and ${ }^{127} \mathrm{I}$ ion beams, aiming at determining the energy loss difference for adjacent $\mathrm{Z}$ values with our set-up, provided also valuable data on the peak shapes in this mass region.

a)

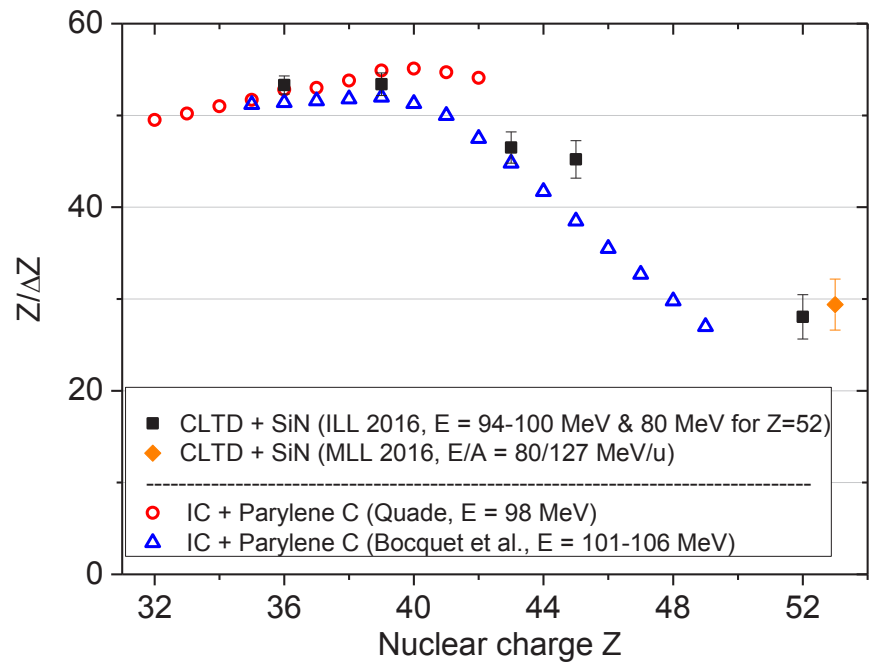

b) Light mass region

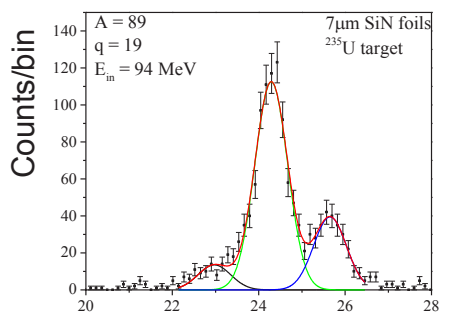

c) Symmetry region

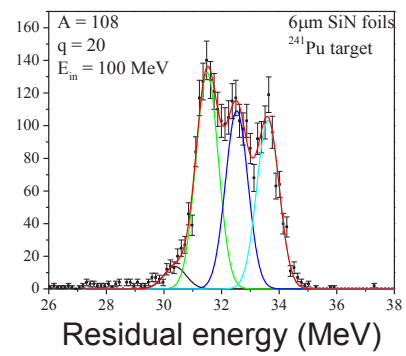

d) Heavy mass region

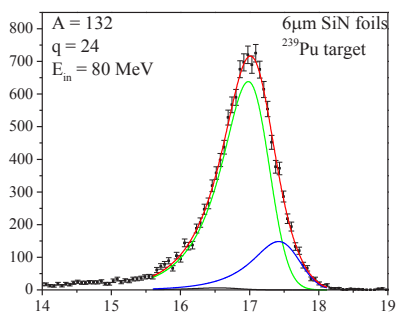

Fig. 5. a) Z-resolving power $(Z / \Delta Z)$ as function of nuclear charge obtained with CLTDs and SiN absorbers for fission fragments at LOHENGRIN and for heavy ions at MLL Garching as compared to data measured with ionization chambers and Parylene-C absorbers by Quade et al. [16] and Bocquet et al. (partly extrapolations) [17]. b), c), d) Sample residual energy spectra for fragments in light, symmetry and heavy mass region respectively where $A$ is the atomic mass, $q$ is the ionic charge state and $E_{\text {in }}$ is the incident energy from LOHENGRIN.

Here, residual energy peaks reveal asymmetric shapes at larger degrader thicknesses, attributed to the influence of energy loss processes. With a precise knowledge on the response function constrained fits for the deconvolution of measured residual energy spectra could be reliably performed, yielding a resolving power of $Z / \Delta Z=28$ for $Z=52$. This demonstrates a significant improvement as compared to extrapolations from previous measurements [17] 
(see Fig. 5a). For the ${ }^{239} \mathrm{Pu}$ target we can also build on recent extensive reference isotopic yield measurements by $\gamma$-ray spectrometry [6]. Besides the possibility of cross-checking available experimental Z-yield data with an alternative technique, we will be able to complete the experimental data sets with masses 130 - 132 and 135, which are either not easily or not at all accessible with $\gamma$-ray spectrometry.

For a precise determination of fractional independent fragment yields with LOHENGRIN it is mandatory to measure systematically nuclear-charge distributions for different ionic charge states $\mathrm{q}$ and kinetic energies $\mathrm{E}$, and follow the target burn-up during the measuring period. Due to its complexity, data analysis for extracting fragment yields is still in progress, and all data shown at present are considered as preliminary. Extended measurements were performed for masses $\mathrm{A}=92$ and 96, for several ionic charges and 5 different energies, and partly for other masses mentioned above. The isotopic fission yields of ${ }^{92} \mathrm{Rb}$ and ${ }^{96} \mathrm{Y}$ from ${ }^{235} \mathrm{U}\left(\mathrm{n}_{\text {th }}, \mathrm{f}\right),{ }^{239} \mathrm{Pu}\left(\mathrm{n}_{\text {th }}, \mathrm{f}\right)$ and ${ }^{241} \mathrm{Pu}\left(\mathrm{n}_{\text {th }}, \mathrm{f}\right)$ were of particular interest, since more precise data than available [21] have been requested recently for achieving a better understanding of the reactor antineutrino spectrum [22,23]. The decay of these isotopes is a main contributor to the integral antineutrino spectra above $4 \mathrm{MeV}$ that are probed by sterile neutrino searches.

\section{Summary and Outlook}

The passive absorber method, for the first time using SiN membranes as degraders and CLTDs as residual-energy detectors, was successfully applied at the LOHENGRIN mass separator at the ILL for the investigation of isotopic yields of fission fragments. A systematic study on Z-resolution with various degrader arrangements was performed in a wide range of fragment masses A and energies E.

Using three fissile targets of ${ }^{235} \mathrm{U},{ }^{239} \mathrm{Pu}$ and ${ }^{241} \mathrm{Pu}$, the quality of nuclear-charge separation was studied for selected masses in the region $82 \leq \mathrm{A} \leq 139$ as a function of degrader thickness and fission-fragment kinetic energies. For the light fragment group, the Z-resolving power attained matches historically best values achieved with Parylene-C absorbers and ionization chambers, while for the symmetry and the heavy mass region a substantial improvement was observed with the new set-up. We have gained first LOHENGRIN data on the isotopic yields in the light-mass group, $89 \leq \mathrm{A} \leq 109$ for ${ }^{241} \mathrm{Pu}$ fission. Towards mass symmetry, known $\mathrm{Z}$-yield data were supplemented in the range $\mathrm{A}=$ 110 to 112 for ${ }^{241} \mathrm{Pu}$, and $\mathrm{A}=111$ to 113 for ${ }^{239} \mathrm{Pu}$, to study odd-even staggering.

Extended data sets were cumulated for the masses $A=92$ and 96 due to special interests in the precise yields of these isotopes for studies on the reactor anti-neutrino spectrum [22,23]. Furthermore, considerable progress was achieved to extend isotopic yield measurements to the heavy-mass region, $128 \leq \mathrm{A} \leq 139$ which was hardly accessible until now.

We finally believe that our approach of deducing isotopic fission fragment yields with applying the novel technologies of CLTDs and SiN degraders still provides a wide scope for further improvements both, from a methodical and technological point of view. 


\section{Acknowledgments}

We would like to thank Norbert Laurens (ILL, Grenoble) for the technical support during the preparation time and the measurement performed at the LOHENGRIN mass separator. This work was supported by the German Research Council (DFG) by means of the Emmy Noether Program and by the German Ministry of Research and Education (BMBF) by grant 05P15RGFAA.

\section{References}

1. Compilation and Evaluation of Fission Yield Nuclear Data, IAEA, Vienna, 2000, IAEA-TECDOC-1168, ISSN 1011-4289; P. Dimitriou, F.-J. Hambsch, and S. Pomp, Fission Product Yields Data, Current status and perspectives, Summary report of an IAEA Technical Meeting IAEA Headquarters, Vienna, 23 -26 May 2016.

2. K.-H. Schmidt, B. Jurado, C. Amouroux, and C. Schmitt, Nuclear Data Sheets 131, 107 (2016).

3. P.Armbruster, M. Asghar, J. P. Bocquet, R. Decker, H. Ewald, J. Grief, E. Moll, B. Pfeiffer, H.Schrader, F. Schussler, G. Siegert, H.Wollnik, Nucl. Instr. Methods 139, 213 (1976).

4. U. Quade, K. Rudolph, G. Siegert, Nucl. Instr. Methods 164, 435 (1979).

5. H.G. Börner and F. Gönnenwein, The Neutron, A Tool and an Object in Nuclear and Particle Physics, World Scientific, Singapore (2012), Chap. 4.

6. O. Serot, IAEA Technical Meeting on Fission Product Yields Data, May 23 - 26, 2016.

7. A.C. Wahl, Atomic Data and Nuclear Data Tables 39, 1 (1988).

8. P. Egelhof, S. Kraft-Bermuth, Top. Appl. Phys. 99, 469 (2005).

9. S. Kraft-Bermuth, V.A. Andrianov, A. Bleile, A. Echler, P. Egelhof, A. Kiseleva, O. Kiselev, H.J. Meier, et al., Rev. Sci. Instr. 80, 103304 (2009).

10. A. Echler, P. Egelhof, P. Grabitz, H. Kettunen, S. Kraft-Bermuth, K. Müller, M. Rossi, W.H. Trzaska, A. Virtanan, J. Low Temp. Phys. 176, 1033 (2014).

11. A. Echler, P. Egelhof, P. Grabitz, H. Kettunen, S. Kraft-Bermuth, K. Müller, M. Rossi, W.H. Trzaska, A. Virtanan, Nucl. Instr. Methods 391, 38 (2017).

12. P. Grabitz, V. Andrianov, S. Bishop, A. Blanc, S. Dubey, A. Echler, P. Egelhof, H. Faust, F. Gönnenwein, J. M. Gomez-Guzman, U. Köster, S. Kraft-Bermuth, M. Mutterer, P. Scholz, S. Stolte, J. Low Temp. Phys. 184, 944 (2016).

13. Supplier: Silson Ltd, Insight Park, Welsh Road East, Southam, Warwickshire, CV47 $1 \mathrm{NE}$, England.

14. M. Döbeli, C. Kottler, M. Stocker, S. Weinmann, H.-A. Synal, M. Grajcar, M. Suter, Nucl. Instr. and Meth. B 219, 415 (2004).

15. Supplier: Attocube Systems AG, Königinstrasse 11a, D-80539 München

16. U. Quade, K. Rudolph, S. Skorka, P. Armbruster, H.G. Clerc, W. Lang, M. Mutterer, C. Schmitt, J.P. Theobald, F. Gönnenwein, J. Pannicke, H. Schrader, G. Siegert, D. Engelhardt, Nucl. Phys. A 487, 1 (1988).

17. J.P. Bocquet, R. Brissot and H.R. Faust, Nucl. Instr. Meth. A 267, 466 (1988). 
18. P. Schillebeeckx, C. Wagemans, P. Geltenbort, F. Gönnenwein, A. Oed, Nucl. Phys. A 580, 15 (1994).

19. D. A. Dwyer, and T.J. Langford, Phys. Rev. Lett. 114, 012502 (2015).

20. M. J. Cubison, and J. L. Jimenez, Atmos. Meas. Tech. 8, 2333 (2015).

21. H.G. Clerc, K.H. Schmidt, H. Wohlfahrth, W. Lang, H. Schrader, K.E. Pferdekämper, R. Jungmann, M. Ashgar, J.P. Bocquet, G. Siegert, Nucl. Phys. A 274, 74 (1974).

22. A.A. Sonzogni, T.D. Johnson, E.A. McCutchan, Phys. Rev. C 91, 011301(R) (2015).

23. F. Reymund and M. Caamano, Seminar on Fission, Corsendonk, World Scientific (2008), p 71. 